\title{
The first confirmed host record of Colletotrichum gloeosporioides on Citrus reticulata subsp. unshiu in the humid subtropics of Russia
}

\section{Padaruth OD ${ }^{1,2}$, Pem $D^{1,2}$, Harishchandra DL ${ }^{1,2,3}$, Jeewon $\mathrm{R}^{4}$, Bulgakov $\mathrm{TS}^{5}$ and Jayawardena $\mathbf{R S}^{1,2}$}

${ }^{1}$ Center of Excellence in Fungal Research, Mae Fah Luang University, Chiang Rai 57100, Thailand

${ }^{2}$ School of Science, Mae Fah Luang University, Chiang Rai 57100, Thailand

${ }^{3}$ Institute of Plant and Environment Protection, Beijing Academy of agriculture and forestry sciences, Beijing 100097, People's Republic of China

${ }^{4}$ Dept of Health Sciences, Faculty of Medicine and Health Sciences, University of Mauritius, Reduit, Mauritius

${ }^{5}$ Department of Plant Protection, Federal Research Centre the Subtropical Scientific Centre of the Russian Academy of Sciences, Yana Fabritiusa Street 2/28, Sochi 354002, Krasnodar Region, Russia

Padaruth OD, Pem D, Harishchandra DL, Jeewon R, Bulgakov TS, Jayawardena RS 2021 - The first confirmed host record of Colletotrichum gloeosporioides on Citrus reticulata subsp. unshiu in the humid subtropics of Russia. Plant Pathology \& Quarantine 11(1), 34-48, Doi $10.5943 / p p q / 11 / 1 / 5$

\begin{abstract}
A fresh collection of Colletotrichum gloeosporioides was recovered from Citrus reticulata Blanco in the humid subtropics of the Krasnodar region (Russia). Morphological examinations were performed and multi-loci phylogenies based on DNA sequences derived from actin (ACT), internal transcribed spacers (ITS), glyceraldehyde-3-phosphate dehydrogenase (GAPDH), chitin synthase (CHS) and $\beta$-tubulin (TUB2) were generated to identify the species and investigate its evolutionary relationships to extant species. Our study provides the first confirmed host record of Colletotrichum gloeosporioides on Citrus reticulata Blanco subsp. unshiu in the humid subtropics of Russia. A brief introduction on Citrus reticulata and the impact of Colletotrichum gloeosporioides are discussed. Illustrations of the new host record are also provided.
\end{abstract}

Keywords - Asexual fungi - Coelomycetes - Garden Museum "Tree of Friendship" - Krasnodar Region - Phylogeny - Satsuma mandarin - Sochi - Taxonomy

\section{Introduction}

Mandarin orange or Citrus reticulata Blanco (Rutaceae) is a spontaneous hybrid that is highly diversified and is an economically important crop (Phetkul et al. 2013, Usman \& Fatima 2018). It has a wide distribution, high production and high demand on the market (Sharma \& Sharma. 2009, Phetkul et al. 2013, Usman \& Fatima 2018). Mandarin is a common fruit in tropical and subtropical regions, especially the Mediterranean region, including its northern-eastern edge the Black Sea Coast of Caucasus within Russia (Sanabam et al. 2015, Raldugina \& Kulyan 2018, Usman \& Fatima 2018).

Citrus fruits confer health benefits as they are rich with physiologically active compounds (Tennant et al. 2009, Ahmed et al. 2020). They consist of phytochemicals that have anti-cancer and 
antifungal properties against species such as Alternaria, Rhizoctonia, Curvularia and Fusarium (Lota et al. 2000, Chutia et al. 2009, Tennant et al. 2009).

Citrus reticulata Blanco subsp. unshiu (Marcov.) D. Rivera Nunez et al. (syn. C. unshiu (Swingle) Marcov.) is a semi-seedless and easy-peeling Citrus species, also known as Satsuma mandarin, unshu mikan, or cold hardy mandarin (Fujii et al. 2016). It was named after its original location Wenzhou (Unsyu in Japanese spelling) in China, but introduced to the West via Japan (Fujii et al. 2016). This host is considered as a separate mandarin species by the Tanaka classification system, but it is treated as a group of mandarin varieties based on the Swingle system (Froelicher et al. 2011). Modern phylogenetic studies have shown the Satsuma mandarin is a highly inbred mandarin-pomelo hybrid (Wu et al. 2018).

Citrus reticulata subsp. unshiu are the most widely grown Citrus in the Black Sea coastal area of the Western Caucasus in Russia and Abkhazia because of their market desirability and winter-hardiness (Ryndin \& Kulyan 2013, Raldugina \& Kulyan 2018, Volk et al. 2018). Largescale plantings of Satsuma mandarins and other Citrus in the Black Sea Coast of Caucasus since the mid-1900s demonstrated that this Citrus could be a profitable crop in the humid subtropics of Abkhazia and Krasnodar region of Southern European Russia and adjacent Abkhazia and Georgia (Kulyan 2013, Ryndin et al. 2014). Citrus production has increased and yields have become more predictable in the Black Sea Coast of Russia since the middle of the $20^{\text {th }}$ century. The breeding of new cold-hardy varieties of Satsuma mandarin has led to a more adaptable Citrus culture to the local conditions of the humid subtropics of the Russian Black Sea Coast for growers (Ryndin et al. 2014, Raldugina \& Kulyan 2018, Volk et al. 2018).

Scientists of the Subtropical Scientific Center of the Russian Academy of Sciences in Sochi (former Russian Research Institute of Floriculture and Subtropical Crops) created new adapted local varieties of Satsuma mandarin and other Citrus species. The Center has the largest Citrus collections in Russia: 132 varieties of several Citrus species: citron ( $C$. medica L.), grapefruit (C. paradisi Macfad.), Ichang papeda (C. cavaleriei H. Lév.), lemon (C. limon (L.) Burm. fil.), mandarin (Citrus reticulata Blanco, including parthenocarpic varieties of Citrus reticulata var. unshiu), pomelo (Citrus grandis (L.) Osbeck), kumquat (Citrus japonica Thunb.), sour orange (Citrus $\times$ aurantium L.), sweet orange (Citrus $\times$ sinensis $(\mathrm{L}$.$) Osbeck), trifoliate orange (Citrus$ trifoliata L.), and yuzu (Citrus junos Sieb. ex Tanaka), which were brought from Japan, USA, Italy, Spain, Nicaragua and Abkhazia (Ryndin \& Kulyan 2016). The unique experimental gardenmuseum "Tree of Friendship" in Sochi is one of the main bases for the research and breeding of Citrus species and their varieties in Russia, including the unique object - Tree of Friendship. This tree was planted by breeder F.M. Zorin in 1934 as a scientific experiment to create a new mandarin hybrid, but soon the Tree became a unique collection of 45 different Citrus species and varieties grafted on this Citrus tree by many famous people from many countries. The tree was officially named "Tree of Friendship" in 1957, and in 1981 the former experimental breeding base around the Tree ( 2 ha) was transformed into a public Garden-Museum "Tree of Friendship" with the special Museum building (Kravtsov 2009).

Low production of Citrus fruits may often be due to unfavorable weather conditions, pests and diseases caused by many microorganisms, especially fungi and viruses (Sharma \& Sharma 2009, Tennant et al. 2009, Usman \& Fatima 2018). The symptoms of the diseases can be observed on the aerial parts of the host such as the leaves, branches and fruits (Sharma \& Sharma 2009). Diseases that are common among Citrus are anthracnose, cankers Citrus gummosis, the decay of fibrous root, crown rot, foot rot, brown rot, necrosis of tissue and canopy blight (Jiang et al. 2012, Naqvi 2004). Different fungal species are the most numerous and significant pathogens of Citrus species in the Black Sea Coast of Russia. Nowadays at least 35 plant pathogenic fungi were registered on Citrus species in the humid subtropics of Krasnodar Region and adjacent Abkhazia (Aiba et al. 2018). The most widespread fungal pathogens among them are Colletotrichum gloeosporioides (Penz.) Penz. \& Sacc. (anthracnose of leaves and twigs). Others, but most devastating pathogens are Plenodomus tracheiphilus (Petri) Gruyter, Aveskamp \& Verkley, Phytophthora citricola Sawada, and Verticillium albo-atrum Reinke \& Berthold, which can infect 
many parts of Citrus trees and ultimately cause the death of the trees (Aiba et al. 2018).

Sometimes, Citrus fruits may be affected by Alternaria alternata (Fr.) Keissl., Aspergillus niger Thiegh., Botrytis cinerea Pers., Elsinoe fawcettii Bitanc. \& Jenkins, Phyllosticta citricarpa (McAlpine) Aa, Trichothecium roseum (Pers.) Link, and several Aspergillus, Fusarium and Penicillium species. Leaf spots could be caused by Mycosphaerella gibelliana (Pass.) Jacz. and Phyllosticta citricola Sacc. and sooty moulds in Citrus leaves by Aithaloderma citri (Briosi \& Pass.) Woron. and Meliola citricola Syd. \& P. Syd. are usual sooty moulds on Citrus leaves (Aiba et al. 2018). Macrophoma mantegazziana (Penz.) Berl. \& Voglino, Lasiodiplodia theobromae (Pat.) Griffon \& Maubl., Nectria cinnabarina (Tode) Fr., Sphaeropsis tumefaciens Hedges, and several Dothiorella spp. cause blight and branch necrosis of Citrus trees, and Citrus roots rot may occur due to Armillaria mellea (Vahl) P. Kumm., Athelia rolfsii (Curzi) C.C. Tu \& Kimbr., Irpex lacteus (Fr.) Fr., Ganoderma applanatum (Pers.) Pat., G. lucidum (Curtis) P. Karst., Sclerotinia sclerotiorum (Lib.) de Bary, and several Phytophthora species cause Citrus root rots (Aiba et al. 2018).

\section{Colletotrichum species and their importance}

Corda (1831) introduced Colletotrichum, the only member of Glomerellaceae (Réblová et al. 2011, Maharachchikumbura et al. 2015, Jayawardena et al. 2016a, Hyde et al. 2020a). The genus was initially accommodated in Phyllachoraceae, and later it was placed in Glomerellaceae in 1984 by Locquin. The placement was validated when phylogenetic data became available and Réblová et al. (2011) clarified the placement (Hyde et al. 2020a). Species in the Colletotrichum are wellknown pathogens on plants and humans (Jayawardena et al. 2017, Bhunjun et al. 2019, Jayawardena et al. 2021). The Species Fungorum database counts over 200 species names for Colletotrichum since August 2020 (http://www.speciesfungorum.org). Colletotrichum species are usually misidentified because of overlapping morphological characteristics and differences in taxonomists' perceptions. Previously, scientists had the misconception that Colletotrichum is hostspecific which resulted in species nomenclatural problems (Farr et al. 2006, Jayawardena et al. 2016a, b). Also, several species lack type specimens of living strains to carry out DNA based identifications (Jayawardena et al. 2016b). DNA based multi-loci phylogenetic analyses have largely improved species identification and this has also led to species synonymy and provided much better taxonomic insights into the naming of species based on hosts (Farr et al. 2006, Lopes da Silva et al. 2019).

Colletotrichum life modes can be saprobic, pathogenic and endophytic. Some Colletotrichum species can change their lifestyle depending on nutritional requirements and environmental conditions (Farr et al. 2006, Nesher et al. 2008, Cannon et al. 2012, Jeewon et al. 2013, Jayawardena et al. 2016a, b, Jeewon et al. 2017, Samarakoon et al. 2018). Conidia and ascospores of Colletotrichum spread with the help of water splashes during rainfalls (Farr et al. 2006, Cannon et al. 2012). Several species of Colletotrichum can cause infection by forming appressorium that will penetrate the host and facilitate germination (Farr et al. 2006, Cannon et al. 2012, Jayawardena et al. 2016a, b).

Highly pathogenic Colletotrichum species are reported to affect several important berries and fruit crops such as avocado (Persea americana), Citrus, banana (Musa spp.), mango (Mangifera indica), strawberry (Fragaria ananassa) and tomato (Solanum lycopersicum) (Cannon et al. 2012, Huang et al. 2013, Guarnaccia et al. 2017) that can cause diseases such as root rot, crown rot, brown blotch and anthracnose diseases (Farr et al. 2006, Cannon et al. 2012, Ahmed et al. 2020). Some examples of pathogenic Colletotrichum species are C. acutatum sensu lato, C. fructicola, C. gloeosporioides, C. nymphaeae and C. viniferum (Cannon et al. 2012, Jayawardena et al. 2017, Hyde et al. 2020b).

Colletotrichum gloeosporioides is also known as a preharvest pathogen, which later can transit to necrotrophs during harvest or post-harvest. Furthermore, pathogenic members of Colletotrichum exist as endophytes and often may be introduced to other countries by plants trading (Farr et al. 2006). This can result in yield loss or destruction of the crops if the infection propagates 
(Jiang et al. 2012, Lopes da Silva et al. 2019). Colletotrichum species can attack the host plant leaves regardless of the stage of plant growth (Jiang et al. 2012).

Colletotrichum gloeosporioides can form lesions on some hosts and are seen as small, circular spots that can be water-soaked and sunken and constantly increasing in size (Jiang et al. 2012). The examples of some high-susceptible crops to pathogenic strains of Colletotrichum are Citrus sinensis, Juglans regia, Litchi chinensis and Manihot esculenta amongst others (Jiang et al. 2012). As studies reported, Colletotrichum gloeosporioides can be the usual plant anthracnose pathogen on apple (Malus domestica), olive (Olea europea), ivy (Hedera taurica), privet (Ligustrum vulgare) and prickly Russian thistle (Kali tragus) in the Republic of Crimea (Dudka et al. 2004) and Krasnodar Region of Russia (Kolomiets et al. 2008). This species is also well-known plant pathogen of many Citrus species in Mediterranian countries: Italy (Aiello et al. 2015), Greece and Spain (Guarnaccia et al. 2017), Portugal (Ramos et al. 2016), Morocco (Benyahia et al. 2003), Algeria (Mahiout et al. 2018), Tunisia (Rhaiem \& Taylor 2016), Turkey (Huseyinov \& Selcuk et al. 2001). This species was recorded on citruses in the western part of the South Caucasus region: Republic of Georgia (Zambettakis \& Dzagania 1986), Abkhazia and Black Sea Coast of Russia (Aiba et al. 2018), however, these records were not confirmed by molecular phylogenetic methods.

\section{Materials \& Methods}

\section{Sample collection, isolation and identification}

Leaves of Citrus reticulata Blanco with spots associated with a microfungus was collected in Khostinsky City District, the Garden Museum "Friendship Tree" (Russia, Krasnodar Region, Sochi, Khostinsky City District). Specimens were observed using a Motic SMZ 168 series dissecting stereo-microscope and morphological structures were examined using a stereomicroscope (Zeiss Discovery v8) fitted with Axio Cam ERc5S and photo-captured with a Leica DM2500 compound microscope attached with a Leica MC190 HD camera. Single spore isolations were carried out and pure cultures were obtained following the method described in Chomnunti et al. (2014) and Senanayake et al. (2020). The cultures were incubated for 10 to 15 days at $25^{\circ} \mathrm{C}$ with frequent observation. Sporulation was observed after 2 weeks, after which fungal morphology characters were examined as described by Cai et al. (2009). Morphological measurements were made using Tarosoft ${ }^{\circledR}$ Image Frame Work program and the images were processed and photo plates were made with Adobe Photoshop CC 2019.

\section{DNA extraction, PCR amplification and sequencing}

Fresh mycelium was collected from the margin of colonies on MEA plates and transferred into $1.5 \mathrm{ml}$ microcentrifuge tubes for genomic DNA extraction. Modified CTAB method was used during Genomic DNA extraction from fresh mycelia described by Guo et al. (2000).

DNA amplification was accomplished using known primer pairs, ITS1/ITS4 to obtain sequence for internal transcribed spacer (ITS) of the rRNA gene (White et al. 1990). GDF/GDR was used for the gene coding for glyceraldehyde-3-phosphate dehydrogenase (GAPDH) (Templeton et al. 1992). And ACT512F/ACT783R was used for actin (ACT) (Carbone \& Kohn 1999). During, polymerase chain reactions (PCR) a total volume of $25 \mu \mathrm{l}$ using PCR mixtures containing $16.2 \mu \mathrm{l}$ of ddH2O, $1 \mu \mathrm{l}$ of each primer, $3.0 \mu \mathrm{l}$ of dNTPs (TaKaRa, China), $2.5 \mu \mathrm{l}$ of 10x Ex-Taq buffer (TaKaRa, China), $1 \mu$ l of genomic DNA, and $0.3 \mu l$ of TaKaRa Ex-Taq DNA polymerase (TaKaRa, China) were used. For PCR amplification, A BIORAD C1000 TouchTM Thermal Cycler was used (Applied Biosystems, Foster City, CA, USA). The PCR conditions were as follows: initial denaturation $95^{\circ} \mathrm{C}$ for $3 \mathrm{~min}, 35$ cycles of denaturation at $95^{\circ} \mathrm{C}$ for $30 \mathrm{~s}$, annealing for $48 \mathrm{~s}$, elongation at $72^{\circ} \mathrm{C}$ for $1 \mathrm{~min}$, and a final extension at $72^{\circ} \mathrm{C}$ for $10 \mathrm{~min}$. The temperature for the annealing step was $59^{\circ} \mathrm{C}$ for ITS, $54^{\circ} \mathrm{C}$ for $\mathrm{GAPDH}$ and $56^{\circ} \mathrm{C}$ for the ACT. To visualize the PCR products, ethidium bromide $(\mathrm{EtBr})$ stain was used on $1 \%$ agarose electrophoresis gels. DNA sequencing of the required genes was done using the same PCR primers by Beijing Biomed Gene Technology Co., Ltd, China. 


\section{Phylogenetic analyses}

Phylogenetic analyses were carried out using a combined dataset of ITS, GAPDH, ACT, CHS-1 and TUB2. Separate ITS, GAPDH, and ACT DNA sequences were subjected to the BLAST search engine of NCBI to verify and select taxa for phylogenetic analyses. Taxa used in the analyses were found from the latest publications (Bhunjun et al. 2019).

BioEdit v. 7.0.5.2 (Hall 1999) was used to merge the single sequence datasets (ITS, GAPDH, ACT, CHS-1 and TUB2) into a concatenated dataset. The phylogenetic analyses were based on maximum likelihood (ML), Bayesian posterior probability (BYPP) and Maximum parsimony analysis (MP). MrModeltest 2.2 (Nylander 2004) was used to select the best-fit nucleotide substitution models under the Akaike information criterion (AIC) for Bayesian analysis. Maximum likelihood analyses were generated using RaxMl-HPC2 on XSEDE (8.2.12) (Stamatakis et al. 2008, Stamatakis 2014) in the CIPRES Science Gateway platform (Miller et al. 2010), using the GTR+I+G model of evolution.

Maximum parsimony analysis was performed using the PAUP1.0b10 software with the heuristic search option 1,000 random replicates (Swofford 2004). Maxtrees were set up to 5000 and branches of zero length were collapsed and all multiple parsimonious trees were saved. Length tree (TL), consistency index (CI), retention index (RI), rescaled consistency index (RC) and homoplasy index $(\mathrm{HI})$ were calculated for trees generated under different optimality criteria. The validness of the most parsimonious trees was calculated by 1000 bootstrap replications resulting from maximum parsimony analysis (Hillis \& Bull 1993). Kishino-Hasegawa tests (KHT) (Kishino \& Hasegawa 1989) were performed to determine whether the trees were significantly different.

Bayesian analysis was carried out using MrBayes v 3.1.2 (Huelsenbeck \& Ronquist 2001) to generate a posterior probability. For $10 \times 10^{6}$ generations, six simultaneous Markov chains were used and trees sampling were done at every $100^{\text {th }}$ generation. The stationary phase was examined by checking the distribution of log-likelihood scores and thus, it was decided, if extra runs were needed to achieve convergence, using the program Tracer 1.4 (Drummond \& Rambaut 2007). To generate a reliable tree, $20 \%$ of generated trees were discarded and the remaining $80 \%$ were used to calculate posterior probabilities for the final tree.

Maximum likelihood bootstrap value (BS) equal to or greater than $50 \%$, Bayesian Posterior Probabilities (BYPP) equal to or greater than 0.90 is given below or above each node. Figtree V.14 was used to visualize the tree (Rambaut 2012) and was edited using Microsoft Office PowerPoint 2016. Facesoffungi numbers are acquired as in Jayasiri et al. (2015). The new sequences generated from this study were submitted to GenBank (Table 1).

\section{Results}

\section{Phylogenetic analyses}

The combined alignment dataset comprised 55 taxa. Colletotrichum boninense )CBS 123755( and Colletotrichum catinaense (CBS 142417) were used as outgroup taxa. The MP dataset had 2111 characters of which 1375 were constant, 288 variable characters were parsimonyuninformative, and 448 characters were counted as a parsimony-informative character. The most parsimonious tree had scores as follows: $\mathrm{TL}=1411, \mathrm{CI}=0.65, \mathrm{RI}=0.76, \mathrm{RC}=0.50, \mathrm{HI}=0.35$.

RAxML analysis yielded a best scoring tree )Fig. 1) with a final ML optimization likelihood value of -10818.124024 . The matrix had 874 distinct alignment patterns, with $18.92 \%$ of undetermined characters or gaps. Estimated base frequencies were as follows: $\mathrm{A}=0.225580 \mathrm{C}=$ $0.302893, \mathrm{G}=0.242537, \mathrm{~T}=0.228990$; substitution rates: $\mathrm{AC}=1.097064, \mathrm{AG}=2.844604, \mathrm{AT}=$ 1.056356, $\mathrm{CG}=0.921213, \mathrm{CT}=4.560703$ and $\mathrm{GT}=1.000000$; gamma distribution shape parameter $\alpha=1.103448$. After $2 \times 10^{6}$ generations, the Bayesian analysis resulted in 10000 trees. The first 1000 trees were discarded as it represented the burn-in phase of the analysis. The remaining 9000 trees were used to calculate posterior probabilities in the majority rule consensus tree. 
The MP tree generated indicates that our strain groups with other Colletotrichum gloeosporioides )with strong bootstrap support 99\% ML and 0.99 BYPP.

Table 1 Taxa used in the phylogenetic analyses and their GenBank accession numbers. A newly generated sequence for Colletotrichum gloeosporioides is indicated in bold red. Ex-type strains are indicated in black bold and type species are indicated by *.

\begin{tabular}{|c|c|c|c|c|c|c|c|c|}
\hline \multirow[t]{2}{*}{ Taxa } & \multirow[t]{2}{*}{ Host } & \multirow[t]{2}{*}{ Location } & \multirow{2}{*}{$\begin{array}{l}\text { Culture } \\
\text { accession No. }\end{array}$} & \multicolumn{5}{|c|}{ GenBank Accession No. } \\
\hline & & & & ITS & GAPDH & ACT & CHS-1 & TUB2 \\
\hline C. aenigma & Persea Americana & Israel & ICMP 18608* & JX010244 & JX010044 & JX009443 & JX009774 & JX010389 \\
\hline C. aeschynomenes & Aeschynomene virginica & Arkansas & ICMP 17673* & JX010176 & JX009930 & JX009483 & JX009799 & JX010392 \\
\hline C. alatae & Dioscorea alata & Rajasthan & ICMP 17919* & JX010190 & JX009990 & JX009471 & JX009837 & JX010383 \\
\hline C. alienum & Malus domestica & $\begin{array}{l}\text { New Zealand } \\
\text { North }\end{array}$ & ICMP 12071* & JX010251 & JX010028 & JX009572 & JX009882 & JX010411 \\
\hline C. aotearoa & Coprosma & $\begin{array}{l}\text { New Zealand } \\
\text { North }\end{array}$ & ICMP 18537* & JX010205 & JX010005 & JX009564 & JX009853 & JX010420 \\
\hline C.artocarpicola & Artocarpus heterophyllus & Thailand & $\begin{array}{l}\text { MFLUCC 18- } \\
1167 *\end{array}$ & MN415991 & MN435568 & MN435570 & MN435569 & MN435567 \\
\hline C. asianum & Coffea arabica & Thailand & ICMP 18580* & FJ972612 & JX010053 & JX009584 & JX009867 & JX010406 \\
\hline C.boninense & Crinum asiaticum & Ogasawara-shoto & CBS 123755* & JQ005153 & JQ005240 & JQ005501 & JQ005327 & JQ005588 \\
\hline C. camelliae & Camellia sinensis & China & $\begin{array}{l}\text { CGMCC } \\
3.14925^{*}\end{array}$ & KJ955081 & KJ954782 & KJ954363 & - & KJ955230 \\
\hline C. catinaense & Citrus reticulata & Italy, Catania & CBS 142417* & KY856400 & KY856224 & KY855971 & KY856136 & KY856482 \\
\hline C. chengpingense & Fragaria $\times$ ananassa & China & $\begin{array}{l}\text { MFLUCC 15- } \\
\text { 0022* }\end{array}$ & KP683152 & KP852469 & KP683093 & KP852449 & KP852490 \\
\hline C. citri-maximae & Citrus maximum & China & AGMy0254* & KX943582 & KX943578 & KX943566 & KX943571 & KX943586 \\
\hline C. conoides & Capsicum annuum & China & CAUG17* & KP890168 & KP890162 & KP890144 & KP890156 & KP890174 \\
\hline C. cordylinicola & Cordyline fruticose & Thailand & ICMP 18579* & JX010226 & JX009975 & HM470235 & JX009864 & JX010440 \\
\hline C.endophytica & Pennisetum purpureum & Thailand & $\begin{array}{l}\text { MFLUCC 13- } \\
\text { 0418* }\end{array}$ & KC633854 & KC832854 & KF306258 & - & - \\
\hline C. fructicola & Coffea arabica & Thailand & ICMP 18581* & JX010165 & JX010033 & FJ907426 & JX009866 & JX010405 \\
\hline C. fructivorum & Vaccinium macrocarpon & New Jersey & Coll1414* & $\mathrm{JX} 145145$ & - & - & - & JX145196 \\
\hline C. gloeosporioides & Citrus bergamia & Greece & CPC 27129 & KY856425 & KY856249 & KY855998 & KY856165 & KY856507 \\
\hline C. gloeosporioides & Citrus sinensis & South Africa & AGMy0026b & KX578790 & KX578774 & - & - & KX578806 \\
\hline C. gloeosporioides & Citrus limon & Vietnam & AGMy0250 & KX578801 & KX578785 & - & - & KX578817 \\
\hline C. gloeosporioides & Citrus sinensis & Italy & CBS 112999* & JX010152 & JX010056 & JX009531 & JX009818 & JX010445 \\
\hline
\end{tabular}


Table 2 Continued.

\begin{tabular}{|c|c|c|c|c|c|c|c|c|}
\hline \multirow[t]{2}{*}{ Taxa } & \multirow[t]{2}{*}{ Host } & \multirow[t]{2}{*}{ Location } & \multirow{2}{*}{$\begin{array}{l}\text { Culture } \\
\text { accession No. }\end{array}$} & \multicolumn{5}{|c|}{ GenBank Accession No. } \\
\hline & & & & ITS & GAPDH & ACT & CHS-1 & TUB2 \\
\hline C. gloeosporioides & Citrus sinensis 'Lanelate' & Spain & CBS 142408 & KY856402 & KY856226 & & KY856142 & KY856484 \\
\hline C. gloeosporioides & Citrus reticulata & Russia & $\begin{array}{l}\text { MFLUCC 20- } \\
0148\end{array}$ & MT947088 & MW192774 & MW192773 & - & - \\
\hline C. gloeosporioides & Citrus limon & Italy & CPC 26373 & KY856406 & KY856230 & KY855979 & KY856146 & KY856488 \\
\hline C. gloeosporioides & Citrus paradise & Italy & CPC 26376 & KY856407 & KY856231 & KY855980 & KY856147 & KY856489 \\
\hline C. gloeosporioides & Citrus limon & Italy & CPC 26381 & KY856408 & KY856232 & KY855981 & KY856148 & KY856490 \\
\hline C.gloeosporioides & Citrus sinensis & Zimbabwe & AGMy0246 & KX578797 & KX578781 & - & - & KX578813 \\
\hline C.gloeosporioides & Citrus sinensis & Brazil & AGMy0229a & KX578796 & KX578780 & - & - & KX578812 \\
\hline C. gloeosporioides & Citrus floridana & Italy & CPC 28155 & KY856442 & KY856266 & KY856015 & KY856182 & KY856524 \\
\hline C. gloeosporioides & Citrus medica & Italy & CPC 26515 & KY856412 & KY856236 & KY855985 & KY856152 & KY856494 \\
\hline C. grevilleae & Grevillea spp & Italy & CBS 132879* & KC297078 & KC297010 & KC296941 & KC296987 & KC297102 \\
\hline C. hebeiense & Vitis vinifera & China & $\begin{array}{l}\text { MFLUCC 13- } \\
\text { 0726* }\end{array}$ & KF156863 & KF377495 & KF377532 & KF289008 & KF288975 \\
\hline C. henanense & Camellia sinensis & China & $\begin{array}{l}\text { CGMCC } \\
\text { 3.17354* }\end{array}$ & KJ955109 & KJ954810 & KM023257 & - & KJ955257 \\
\hline C. horii & Diospyros kaki & Japan & ICMP 10492* & GQ329690 & GQ329681 & JX009438 & JX009752 & JX010450 \\
\hline C. hystricis & Citrus hystrix & Italy & СРC 28153 * & KY856450 & KY856274 & KY856023 & KY856190 & KY856532 \\
\hline C. jiangxiense & Camellia sinensis & China & $\begin{array}{l}\text { CGMCC } \\
\text { 3.17363* }\end{array}$ & KJ955201 & KJ954902 & KJ954471 & - & KJ955348 \\
\hline C. kahawae & Coffea arabica & Kenya & ICMP 17816* & JX010231 & JX010012 & JX009452 & JX009813 & JX010444 \\
\hline C. ledongense & Hevea brasiliensis & China & LD1680* & MG242008 & MG242016 & MG242014 & - & KX893580 \\
\hline C. musae & Musa spp. & USA & ICMP 19119* & JX010146 & JX010050 & JX009433 & JX009896 & HQ596280 \\
\hline C. nupharicola & Nuphar lutea & Washington & ICMP 18187 * & JX010187 & JX009972 & JX009437 & JX009835 & JX010398 \\
\hline C.pandanicola & Pandanus spp. & Thailand & $\begin{array}{l}\text { MFLUCC 17- } \\
\text { 0571* }\end{array}$ & MG646967 & MG646934 & MG646938 & MG646931 & MG646926 \\
\hline C. proteae & Protea spp. & South Africa & CBS 132882* & KC297079 & KC297009 & KC296940 & KC296986 & KC297101 \\
\hline C. pseudotheobromicola & Vitis spp. & China & JZB330119* & MG763975 & MG812553 & MG812544 & - & MG812559 \\
\hline C.psidii & Psidium spp. & Italy & ICMP 19120* & JX010219 & JX009967 & JX009515 & JX009901 & JX010443 \\
\hline C. queenslandicum & Carica papaya & Australia & ICMP 1778* & JX010276 & JX009934 & JX009447 & JX009899 & JX010414 \\
\hline C. rhexiae & Rhexia virginica & Delaware & Coll1026* & JX145128 & - & - & - & JX145179 \\
\hline C. salsolae & Salsola tragus & Hungary & ICMP 19051* & JX010242 & JX009916 & JX009562 & JX009863 & JX010403 \\
\hline C. siamense & Coffea arabica & Thailand & ICMP 18578* & JX010171 & JX009924 & FJ907423 & JX009865 & JX010404 \\
\hline C. syzygicola & Syzygium samarangense & Thailand & $\begin{array}{l}\text { MFLUCC 10- } \\
\text { 0624* }\end{array}$ & KF242094 & KF242156 & KF157801 & - & KF254880 \\
\hline C. temperatum & Vaccinium macrocarpon & New York & Coll883* & JX145159 & - & - & - & JX145211 \\
\hline
\end{tabular}


Table 3 Continued.

\begin{tabular}{|c|c|c|c|c|c|c|c|c|}
\hline \multirow[t]{2}{*}{ Taxa } & \multirow[t]{2}{*}{ Host } & \multirow[t]{2}{*}{ Location } & \multirow{2}{*}{$\begin{array}{l}\text { Culture } \\
\text { accession No. }\end{array}$} & \multicolumn{5}{|c|}{ GenBank Accession No. } \\
\hline & & & & ITS & GAPDH & ACT & CHS-1 & TUB2 \\
\hline C. theobromicola & Theobroma cacao & Panama & ICMP 18649* & JX010294 & JX010006 & JX009444 & JX009869 & JX010447 \\
\hline C. $t i$ & Cordyline spp. & New Zealand & ICMP 4832* & JX010269 & JX009952 & JX009520 & JX009898 & JX010442 \\
\hline C. tropicale & Theobroma cacao & Panama & ICMP 18653* & JX010264 & JX010007 & JX009489 & JX009870 & JX010407 \\
\hline C. viniferum & Vitis $\mathrm{p}$ & China & GZAAS5.08601* & JN412804 & JN412798 & JN412795 & - & JN412813 \\
\hline C. wuxiense & Camellia sinensis & China & $\begin{array}{l}\text { CGMCC } \\
\text { 3.17894* }\end{array}$ & KU251591 & KU252045 & KU251672 & KU251939 & KU252200 \\
\hline C. xanthorrhoeae & Xanthorrhoea preissii & Australia & ICMP 17903* & JX010261 & JX009927 & - & JX009823 & JX010448 \\
\hline
\end{tabular}

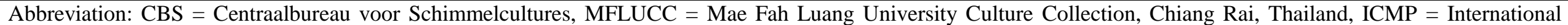
Collection of Microorganisms from Plants, $\mathrm{CGMCC}=$ China General Microbiological Culture Collection Center

Colletotrichum gloeosporioides (Penz.) Penz. \& Sacc., Atti Inst. Veneto Sci. lett., ed Arti, Sér. 6 2(5): 670 (1884)

$\equiv$ Vermicularia gloeosporioides Penz., in Saccardo, Michelia 2 (no. 8): 450 (1882)

Index Fungorum number: IF158410; Facesoffungi number: FoF 09424

Saprobic on Citrus reticulata (Rutaceae) leaves. Sexual morph: undetermined. Asexual morph: Black conidiomata observed in the culture from the centre of the edge. Conidiomata $155-182 \times 232-378 \mu \mathrm{m}(\bar{x}=166 \times 285 \mu \mathrm{m}, \mathrm{n}=6)$ abundant, pycnidial, wide ostiole. Conidiophores $2-3 \times 12-30$ $\mu \mathrm{m}(\bar{x}=2 \times 21 \mu \mathrm{m}, \mathrm{n}=2)$ reduced to conidiogenous cell, hyaline and smooth. Conidiogenous cells $10-39 \mu \mathrm{m}(\bar{x}=21, \mathrm{n}=6)$ hyaline, smooth wall, aseptate, hyaline, slightly rounded at the centre or cylindrical, rounded at the apex and base and guttulated or granular, at times annelids. Appressoria and chlamydospores not observed.

Culture Characteristics - Colonies growing from single conidia on MEA plates white mycelium. Grey in the centre and mycelium changes to white in periphery. The culture reached its maximum diameter $(80 \mathrm{~mm})$ in 14 days at $22^{\circ} \mathrm{C}$. White mycelium, grey to white cottony mycelium, conidial masses developed from orange to black, being black in the centre and white in the periphery. After 14 days, conidia masses could be observed and conidiomata formation was seen after 20 days.

Material examined - RUSSIA, Krasnodar Region, Sochi, Khostinsky City District, Garden Museum "Friendship Tree", on leaves of Citrus reticulata Blanco subsp. unshiu (Marcow.) D. Rivera Nunez et al., 04 August 2018, Timur S. Bulgakov, T-7269, MFLUCC 20-0148 living culture.

Notes - The collected specimen morphologically resembles Colletotrichum gloeosporioides with hyaline spores measuring 10-39 $\mu$ m. Based on, phylogenetic analyses, our strain grouped with all Colletotrichum gloeosporioides species with strong bootstrap support (99\% and 0.99 BYPP). Upon pairwise alignment of nucleotides, no base pair differences were observed in ITS and ACT regions and hence, we identify it as $C$. gloeosporioides based on the recommendations of Jeewon and Hyde (2016). Our species was also recognized as the first record on Citrus reticulata in Russia. 


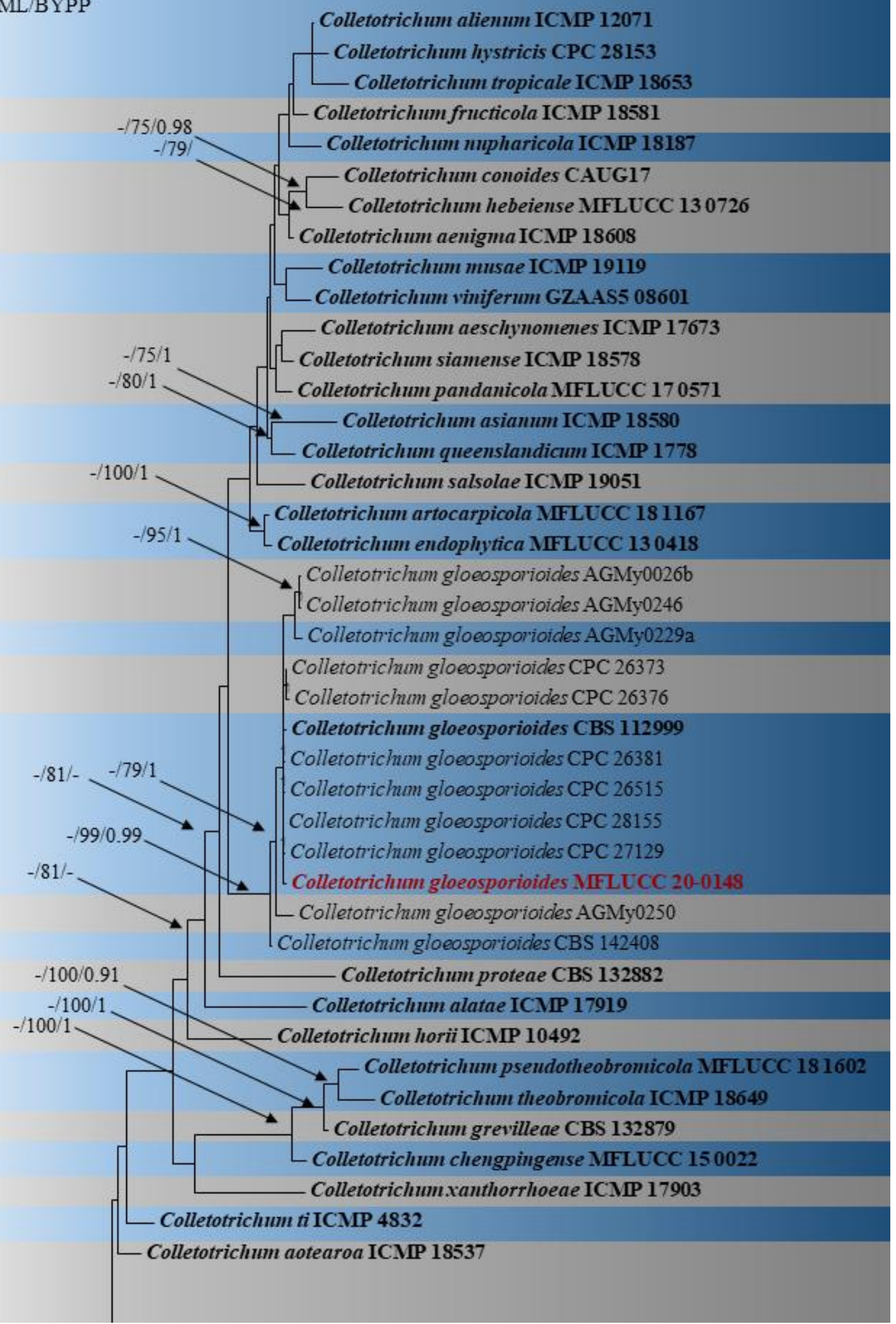

Fig. 1 - Phylogram generated from maximum parsimony (MP) analysis of combined ITS, GAPDH, ACT, CHS and TUB2 sequence data of Colletotrichum. Bootstrap (MP/ML) support values greater than or equal to $70 \%$ are given above the nodes and for BYPP greater than $0.90 \%$. The culture accession number is given along with the species name, and the tree is rooted with $C$. boninense (CBS 123755) and C. catinaense (CBS 142417). The strain obtained in this study is in red bold and ex-types strains are in black bold. 


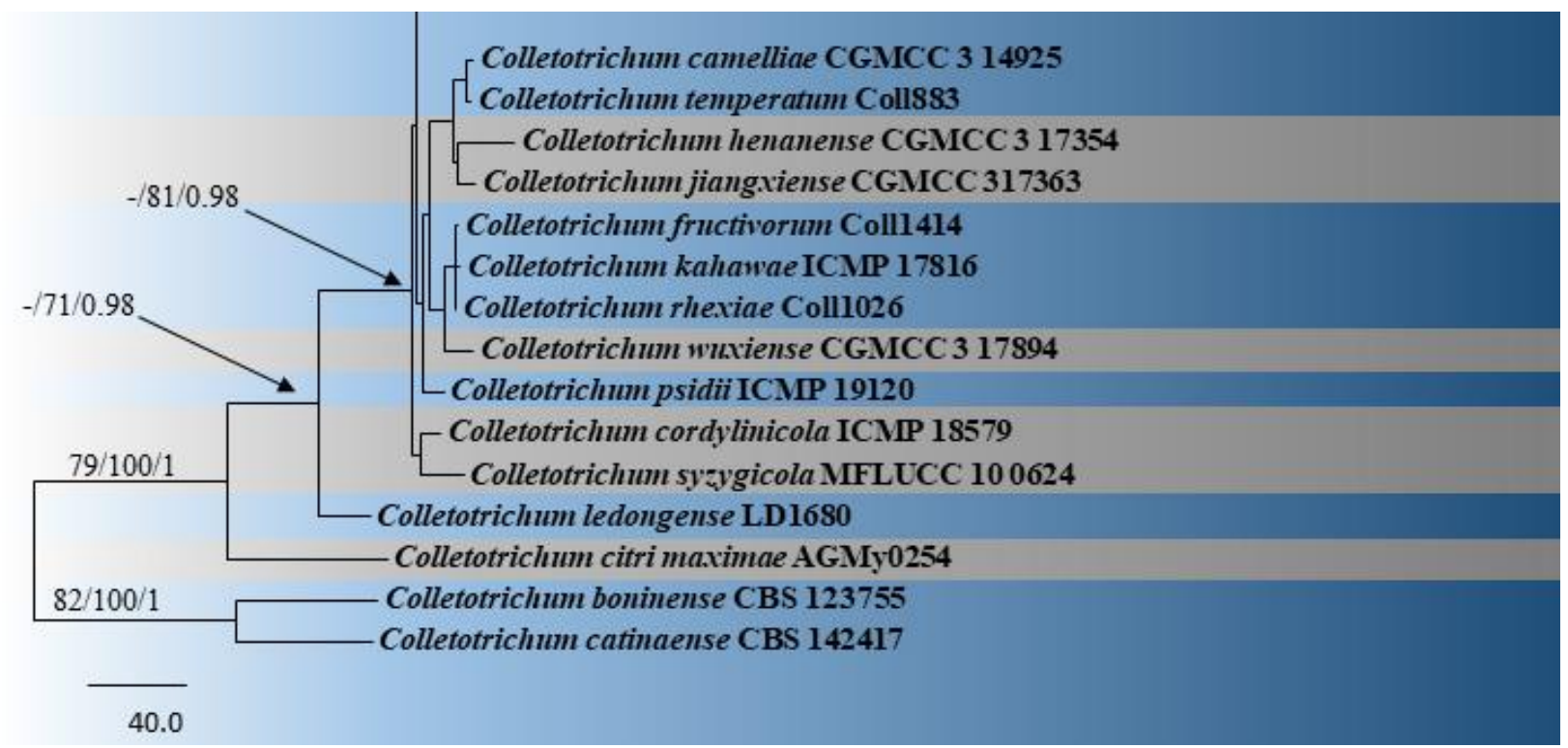

Fig. 1 - Continued.

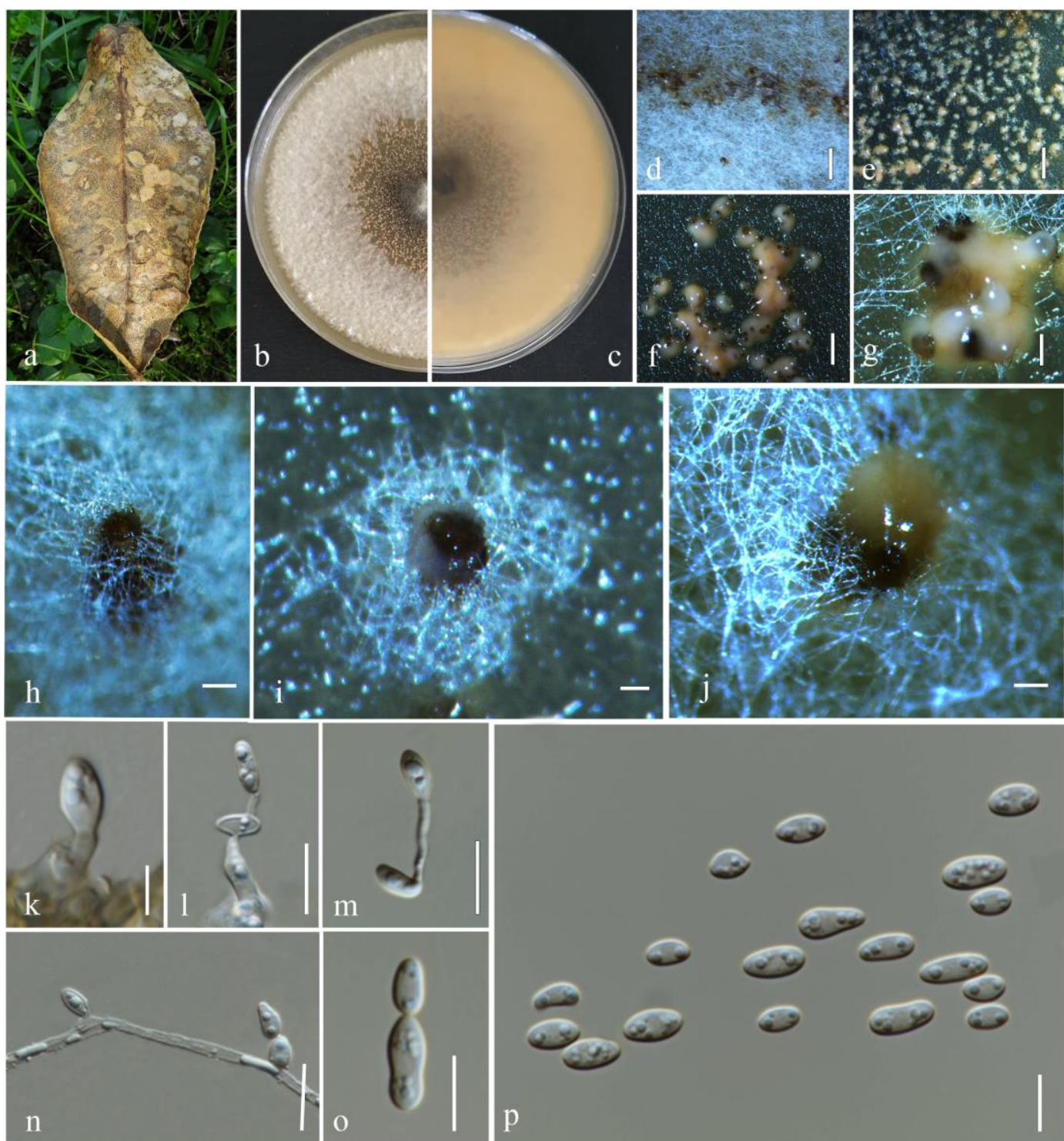

Fig. 2 - Colletotrichum gloeosporioides (MFLUCC 20-0148). a Specimen. b-c Culture on MEA. $\mathrm{a}=$ above view, $\mathrm{b}=$ reverse view $(\mathrm{d}-\mathrm{g}$ Spore mass. $\mathrm{h}-\mathrm{j}$ mature conidiomata. $\mathrm{k}$ conidiophores. 
$1-n$ conidiogenous cells and conidiogenesis. $\mathrm{o}-\mathrm{p}$ conidia. Scale bars: $\mathrm{d}-\mathrm{h}=80 \mu \mathrm{m}, \mathrm{i}-\mathrm{j}=100 \mu \mathrm{m}$, $\mathrm{k}=5 \mu \mathrm{m}, \mathrm{l}=10 \mu \mathrm{m}, \mathrm{m}=15 \mu \mathrm{m}, \mathrm{n}=100 \mu \mathrm{m}, \mathrm{o}=20 \mu \mathrm{m}, \mathrm{p}=100 \mu \mathrm{m}$

\section{Discussion}

Colletotrichum gloeosporioides is known to inhabit several hosts in tropical and sub-tropical countries (Sanders \& Korsten 2003, Rampersad et al. 2013, Douanla-Meli \& Unger 2017, Guarnaccia et al. 2017, Fuentes-Aragón et al. 2018, Tovar-Pedraza et al. 2019, Sakthivel et al. 2020, Satapathy \& Beura 2020). This indicates that Colletotrichum gloeosporioides is resistant to climatic variations (Douanla-Meli \& Unger 2017, Guarnaccia et al. 2017). Since the identification of Colletotrichum is complex, it is important to carry out more sampling from Citrus hosts and on a wider geographical scale. This will help future research studies to identify locations that are at risk.

This is the first confirmed record of Colletotrichum gloeosporioides on Citrus reticulata Blanco in the humid subtropics of Russia, and it is worth noting that this fungal pathogen is already known in the Black Sea Coast of the Caucasus, where it causes anthracnose of many plants, including cultivated Citrus spp. (Aiba et al. 2018).

\section{Acknowledgements}

The authors express their gratitude to the Garden Museum "Tree of Friendship" (Russia) and Zhongkai University of Agriculture and Engineering (China) for facilitating the research. The research was done as part of the implementation of the State Assignment No. 0492-2021-0007 of the Federal Research Centre the Subtropical Scientific Centre of the Russian Academy of Sciences. Dr Rajesh Jeewon would like to thank the University of Mauritius and Mauritius Research and Innovation Council (MRS/RUN-1705) for research support. RS Jayawardena thanks the National Research Council of Thailand, a grant for new researcher NRCT5-TRG630010-01, entitled "Biodiversity, taxonomy, phylogeny and evolution of Colletotrichum in northern Thailand".

\section{References}

Ahmed T, Muqit A, Datta J, Haque M, Haque M. 2020 - Prevalence and severity of different Citrus diseases in Sylhet region. Journal of Bioscience and Agriculture Research 23, 19571968.

Aiba LYa, Karpun NN, Ignatova EA, Shinkuba MS et al. 2018 - Atlas of pests and diseases of Citrus crops in humid subtropics of the Black-Sea coast of the Caucasus. Sochi-Sukhum, 1205.

Aiello D, Carrieri R, Guarnaccia V, Vitale A et al. 2015 - Characterization and pathogenicity of Colletotrichum gloeosporioides and C. karstii causing preharvest disease on Citrus sinensis in Italy. Journal of Phytopathology. 163(3): 168-177.

Benyahia H, Jrifi A, Smaili C, Afellah M, Timmer LW. 2003 - First report of Colletotrichum gloeosporioides causing withertip on twigs and tear stain on fruit of Citrus in Morocco. New Disease Reports 7: 20.

Bhunjun C, Jayawardena R, Wei D, Huanraluek N et al. 2019 - Multigene phylogenetic characterisation of Colletotrichum artocarpicola sp. nov. from Artocarpus heterophyllus in northern Thailand strong. Phytotaxa, 418, 273-286.

Cai L, Hyde KD, Taylor PWJ, Weir BS et al. 2009 - A polyphasic approach for studying Colletotrichum. Fungal Diversity 39, 183-204.

Cannon P, Damm U, Johnston P, Weir B. 2012 - Colletotrichum - Current status and future directions. Studies in Mycology 73, 181-213.

Carbone I, Kohn L. 1999 - A Method for Designing Primer Sets for Speciation Studies in Filamentous Ascomycetes. Mycologia 9, 553.

Chomnunti P, Hongsanan S, Aguirre-Hudson B, Tian Q et al. 2014 - The sooty moulds. Fungal Diversity 66, 1-36. 
Chutia M, Deka Bhuyan P, Pathak MG, Sarma TC et al. 2009 - Antifungal activity and chemical composition of Citrus reticulata Blanco essential oil against phytopathogens from North East India. LWT -Food Science and Technology 3, 777-780.

Douanla-Meli C, Unger J. 2017 - Phylogenetic study of the Colletotrichum species on imported Citrus fruits uncovers a low diversity and a new species in the Colletotrichum gigasporum complex. Fungal Biology 121, 858-868.

Drummond AJ, Rambaut A. 2007 - BEAST: Bayesian evolutionary analysis by sampling trees. BMC Evolutionary Biology 7, 214.

Dudka IO, Heluta VP, Tykhonenko YY, Andrianova TV et al. 2004 - Fungi of the Crimean Peninsula. M.G. Kholodny Institute of Botany, National Academy of Sciences of Ukraine, 452 p. (in Ukrainian)

Farr D, Aime M, Rossman, Palm M. 2006 - Species of Colletotrichum on Agavaceae. Mycological Research 110, 1395-1408.

Froelicher Y, Mouhaya W, Bassene JB, Costantino G et al. 2011 - New universal mitochondrial PCR markers reveal new information on maternal Citrus phylogeny. Tree Genetics \& Genomes, 7(1), 49-61.

Fuentes-Aragón D, Juárez-Vázquez S, Vargas-Hernández M, Silva-Rojas H. 2018 - Colletotrichum fructicola, a Member of Colletotrichum gloeosporioides sensu lato, is the Causal Agent of Anthracnose and Soft Rot in Avocado Fruits cv. "Hass". Mycobiology 46, 92-100.

Fujii H, Ohta S, Nonaka K, Katayose Y et al. 2016 - Parental diagnosis of satsuma mandarin (Citrus unshiu Marc.) revealed by nuclear and cytoplasmic markers. Breeding Science, 16060.

Guarnaccia V, Groenewald J, Polizzi G, Crous P. 2017 - High species diversity in Colletotrichum associated with Citrus diseases in Europe. Persoonia - Molecular Phylogeny and Evolution of Fungi 39, 32-50.

Guo LD, Hyde KD, Liew ECY. 2000 - Identification of endophytic fungi from Livistona chinensis based on morphology and rDNA sequences. New Phytologist 147, 617-630.

Hall TA. 1999 - BioEdit: a user-friendly biological sequence alignment editor and analysis program for Windows 95/98/NT. In: Nucleic Acids. Symposium Series 41, 95-98.

Hillis DM, Bull JJ. 1993 - An empirical test of bootstrapping as a method for assessing confidence in phylogenetic analysis. Systematic Biology 42, 182-192.

Huang F, Chen GQ, Hou X, Fu YS et al. 2013 - Colletotrichum species associated with cultivated Citrus in China. Fungal Diversity, 61(1), 61-74.

Huelsenbeck JP, Ronquist F. 2001 - MRBAYES: Bayesian inference of phylogenetic trees. Bioinformatics 17, 754-755.

Huseyinov E, Selcuk F. 2001 - Contribution to study of mycoflora of Turkey I. Coelomycetes of orders Melanconiales and Sphaeropsidales on forest trees and shrubs in the Black Sea coast (Rize and Trabzon Provinces). Mikologia i Fitopatologia. 35(1), 28-33.

Hyde K, Norphanphoun C, Maharachchikumbura S, Bhat D et al. 2020a - Refined families of Sordariomycetes. Mycosphere, 11, 305-1059.

Hyde KD, de Silva NI, Jeewon R, Bhat DJ et al. 2020b - AJOM new records and collections of fungi: 1-100. Asian Journal of Mycology, 3(1), 22-294.

Jayasiri S, Hyde K, Ariyawansa H, Bhat J et al. 2015 - The Faces of Fungi database: fungal names linked with morphology, phylogeny and human impacts. Fungal Diversity 74, 3-18.

Jayawardena R, Camporesi E, Elgorban A, Bahkali A et al. 2017 - A new species of Colletotrichum from Sonchus sp. in Italy. Phytotaxa 314, 55.

Jayawardena R, Huang J, Jin B, Yan J et al. 2016a - An account of Colletotrichum species associated with strawberry anthracnose in China based on morphology and molecular data. Mycosphere, 7, 1147-1163.

Jayawardena RS, Hyde KD, Damm U, Cai L et al. 2016b - Notes on currently accepted species of Colletotrichum. Mycosphere 7, 1192-1260. 
Jayawardena RS, Bhunjun CS, Hyde KD, Gentekaki E. 2021 - Colletotrichum: lifestyles, biology, morpho-species, species complexes and accepted species. Mycosphere 12, 519-669

Jeewon R, Hyde KD. 2016 - Establishing species boundaries and new taxa among fungi: Recommendations to resolve taxonomic ambiguities. Mycosphere 7, 1669-1677.

Jeewon R, Ittoo J, Mahadeb D, Jaufeerally-Fakim Y et al. 2013 - DNA based identification and phylogenetic characterisation of endophytic and saprobic fungi from Antidesma madagascariense, a medicinal plant in Mauritius. Journal of Mycology

Jeewon R, Wanasinghe DN, Rampadaruth S, Puchooa D et al. 2017 - Nomenclatural and identification pitfalls of endophytic mycota based on DNA sequence analyses of ribosomal and protein genes phylogenetic markers: A taxonomic dead end? Mycosphere 8, 1802-1817.

Jiang Y, Tan P, Zhou X, Hou X et al. 2012 - Colletotrichum gloeosporioides, the causal agent of citrus anthracnose in Guizhou Province. Plant Pathology \& Quarantine 2, 25-29.

Kishino H, Hasegawa M. 1989 - Evaluation of the maximum likelihood estimate of the evolutionary tree topologies from DNA sequence data, and the branching order in hominoidea. Journal of Molecular Evolution 29(2), 170-179.

Kolomiets T, Skatenok O, Alexandrova A, Mukhina Z et al. 2008 - First report of anthracnose of Salsola tragus caused by Colletotrichum gloeosporioides in Russia. Plant disease, 92(9), 1366-1366.

Kravtsov IA. 2009 - History of the creation of the experimental collection of Garden-Museum "Tree of Frendship". Horticulture and Viticulture 6, 6-9. (in Russian)

Kulyan RV. 2013 - Selection of Citrus fruits (tangerines) in the subtropical zone of the Krasnodar region. Horticulture and Viticulture 2, 11-16. (in Russian)

Lopes da Silva L, Dórea Bragança C, Fortes Ferreira C, Alves Silva Diamantino et al. 2019 Differentiation of lineages within "Colletotrichum gloeosporioides s.l." associated with cassava anthracnose disease by BOX- and ERIC-PCRs. Journal of Phytopathology 167, 218 229.

Lota ML, De Rocca Serra D, Tomi F, Joseph Casanova. 2000 - Chemical variability of peel and leaf essential oils of mandarins from Citrus reticulata Blanco. Biochemical Systematics and Ecology 28, 61-78.

Maharachchikumbura SSN, Hyde KD, Jones EBG, McKenzie EHC et al. 2015 - Towards a natural classification and backbone tree for Sordariomycetes. Fungal Diversity 72, 199-301.

Mahiout D, Bendahmane BS, Youcef Benkada M, Mekouar H et al. 2018 - First report of Colletotrichum gloeosporioides on Citrus in Algeria. Phytopathologia Mediterranica. 57(2), 355-359.

Miller M, Pfeiffer W, Schwartz T. 2010 - Creating the CIPRES Science Gateway for inference of large phylogenetic trees. Gateway Computing Environments Workshop )GCE(, 1-10.

Naqvi SAMH. 2004 - Diagnosis and Management of Certain Important Fungal Diseases of Citrus. Diseases of Fruits and Vegetables Volume I, 247-290.

Nesher I, Barhoom S, Sharon A. 2008 - Cell cycle and cell death are not necessary for appressorium formation and plant infection in the fungal plant pathogen Colletotrichum gloeosporioides. BMC Biology 6, 9.

Nylander JAA. 2004 - MrModeltest Version 2. Program Distributed by the Author. Uppsala: Evolutionary Biology Centre, Uppsala University.

Phetkul U, Wanlaso N, Mahabusarakam W, Phongpaichit S et al. 2013 - New acridone from the wood of Citrus reticulata Blanco. Natural Product Research 27, 1922-1926.

Raldugina SN, Kulyan RV. 2018 - A brief overview of producing fruits from mandarin group (mandarin, tangerine, clementine) in some countries across the World. Subtropical and ornamental Horticulture 64, 19-25. (in Russian)

Rambaut A. 2012 - FigTree v1.4: Tree figure drawing tool. http://tree bio.ed.ac.uk/software/ figtree/

Ramos AP, Talhinhas P, Sreenivasaprasad S, Oliveira H. 2016 - Characterization of Colletotrichum gloeosporioides, as the main causal agent of Citrus anthracnose, and C. karstii 
as species preferentially associated with lemon twig dieback in Portugal. Phytoparasitica 44(4), $549-561$

Rampersad SN, Perez-Brito D, Torres-Calzada C, Tapia-Tussell R et al. 2013 - Genetic structure and demographic history of Colletotrichum gloeosporioides sensu lato and C. truncatum isolates from Trinidad and Mexico. BMC Evolutionary Biology 131, 130.

Réblová M, Gams W, Seifert KA. 2011 - Monilochaetes and allied genera of the Glomerellales, and a reconsideration of families in the Microascales. Studies in Mycology 68, 163-191.

Rhaiem A, Taylor PWJ. 2016 - Colletotrichum gloeosporioides associated with anthracnose symptoms on Citrus, a new report for Tunisia. European Journal of Plant Pathology 146, 219-224

Ryndin AV, Belous OG, Malyarovskaya VI, Pritula ZV et al. 2014 - Physiological and biochemical techniques for studying adaptation mechanisms of subtropical, southern fruit and ornamental crops in Russian subtropics. Agricultural Biology 3, 40-48. (in Russian).

Ryndin AV, Kulyan RV. 2013 - Genetic potential of modern Citrus assortment in the Black Sea Coast of Russia. Herald of Russian Academy of Agricultural Sciences 6, 41-45. (in Russian).

Ryndin AV, Kulyan RV. 2016 - Collection of Citrus cultures in the humid subtropics of Russian Federation Horticulture and Viticulture 5, 24-30. (in Russian).

Sakthivel K, Sawhney S, Manigundan K, Pandey VK et al. 2020 - Variability Colletotrichum infecting chilli plants Andaman and Nicobar Island, India. Journal of Environmental Biology 41, 261-265.

Samarakoon MC, Peršoh D, Hyde KD, Bulgakov TS et al. 2018 - Colletotrichum acidae sp. nov. from northern Thailand and a new record of $C$. dematium on Iris sp. Mycosphere 9, 583-597.

Sanders G, Korsten L. 2003 - A comparative morphological study of South African avocado and mango isolates of Colletotrichum gloeosporioides. Canadian Journal of Botany 81, 877-885.

Sanabam R, Singh N, Handique P, Devi H. 2015 - Disease-free khasi mandarin )Citrus reticulata Blanco( production using in vitro microshoot tip grafting and its assessment using DASELISA and RT-PCR. Scientia Horticulturae 189, 208-213.

Satapathy R, Beura S. 2018 - Management of Colletotrichum gloeosporioides (Penz.) Causing Cashew Anthracnose through Botanicals. International Journal of Current Microbiology and Applied Sciences 7, 3539-3543.

Senanayake I, Rathnayaka A, Marasinghe D, Calabon M et al. 2020 - Morphological approaches in studying fungi: collection, examination, isolation, sporulation and preservation. Mycosphere $11,2678-2754$

Sharma S, Sharma R. 2009 - Citrus Canker Approaching Century: A Review. Tree and forestry science and biotechnology 3, 56-65.

Stamatakis A. 2014 - RAxML version 8: A tool for phylogenetic analysis and post-analysis of large phylogenies. Bioinformatics 30, 1312-1313.

Stamatakis A, Hoover P, Rougemont J. 2008 - A Rapid Bootstrap Algorithm for the RAxML Web Servers. Systematic Biology 57, 758-771.

Swofford DL. 2004 - PAUP: phylogenetic analysis using parsimony, version 4.0 b10. Sinauer Associates, Sunderland.

Templeton M, Rikkerink E, Solon S, Crowhurst R. 1992 - Cloning and molecular characterization of the glyceraldehyde-3-phosphate dehydrogenase-encoding gene and cDNA from the plant pathogenic fungus Glomerella cingulata. Gene 122, 225-23.

Tennant P, Dwight R, Fisher L, Bennett S et al. 2009 - Diseases and Pest of Citrus )Citrus sp.(. Tree and Forestry Science and Biotechnology 3, 81-107.

Tovar-Pedraza J, Mora-Aguilera J, Nava-Díaz C, Lima N et al. 2020 - Distribution and Pathogenicity of Colletotrichum Species Associated with Mango Anthracnose in Mexico. Plant Disease 104, 137-146.

Usman M, Fatima B. 2018 - Mandarin )Citrus reticulata Blanco( Breeding. Advances in Plant Breeding Strategies: Fruits, 465-533. 
Volk G, Samarina L, Kulyan R, Gorshkov V et al. 2018 - Citrus genebank collections: international collaboration opportunities between the US and Russia. Genetic resources and crop evolution, 65(2), 433-447.

White T, Bruns T, Lee S, Taylor J. 1990 - Amplification and direct sequencing if fungal ribosomal RNA genes for phylogenetics. PCR protocols, 315-322.

Wu GA, Terol J, Ibanez V, López-García A et al. 2018 - Genomics of the origin and evolution of Citrus. Nature, 554(7692), 311-316.

Zambettakis C, Dzagania A. 1986 - Comparaison des caracteres morphologiques entre micromycetes phytopathogenes des regions subtropicales (Georgie) et Mediterraneennes. Bull. Soc. Mycol. France 102, 141-154. (in French) 